\title{
Solagigasbacteria: Lone genomic giants among the uncultured bacterial phyla
}

4
Eric D. Becraft ${ }^{1}$, Tanja Woyke ${ }^{2}$, Jessica Jarett ${ }^{2}$, Natalia Ivanova ${ }^{2}$, Filipa Godoy Vitorino ${ }^{3}$, Nicole Poulton ${ }^{1}$, Julia M. Brown ${ }^{1}$, Joseph Brown ${ }^{1}$, C.Y.M. Lau ${ }^{4}$, Tullis Onstott ${ }^{4}$, Jonathan A. Eisen $^{5}$, Duane Moser ${ }^{6}$, Ramunas Stepanauskas ${ }^{1}$

${ }^{1}$ Bigelow Laboratory for Ocean Sciences, East Boothbay, ME, USA, ${ }^{2}$ Joint Genome Institute, Walnut Creek, CA, USA, ${ }^{3}$ American University of Puerto Rico, San Juan, Puerto Rico, ${ }^{4}$ Princeton University, Princeton, NJ, USA, ${ }^{5}$ University of California Davis, Genome Center, Davis, CA, USA. ${ }^{6}$ Desert Research Institute, Las Vegas, NV, USA

Corresponding author:

Ramunas Stepanauskas

60 Bigelow Dr.

East Boothbay, ME 04544

207-315-2567 ext. 308

rstepanauskas@bigelow.org

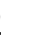


ABSTRACT

Recent advances in single-cell genomic and metagenomic techniques have facilitated the

35

8

9

0

1
discovery of numerous previously unknown, deep branches of the tree of life that lack cultured representatives. Many of these candidate phyla are composed of microorganisms with minimalistic, streamlined genomes lacking some core metabolic pathways, which may contribute to their resistance to growth in pure culture. Here we analyzed single-cell genomes and metagenome bins to show that the "Candidate phylum SPAM" represents an interesting exception, by having large genomes (6-8 Mbps), high GC content (66\%-71\%), and the potential for a versatile, mixotrophic metabolism. We also observed an unusually high genomic heterogeneity among individual SPAM cells in the studied samples. These features may have contributed to the limited recovery of sequences of this candidate phylum in prior metagenomic studies. Based on these observations, we propose renaming SPAM to "Candidate phylum Solagigasbacteria". Current evidence suggests that Solagigasbacteria are distributed globally in diverse terrestrial ecosystems, including soils, the rhizosphere, volcanic mud, oil wells, aquifers and the deep subsurface, with no reports from marine environments to date. 


\section{INTRODUCTION}

Technological innovations in single-cell genomics and metagenomics have led to a rapid improvement in our understanding of the genomic features, evolutionary histories and metabolic capabilities of tens of phylum-level branches of Archaea, Bacteria and Eukarya that lack cultured representatives (Yoon et al., 2011; Rinke et al., 2013; Becraft et al., 2015; Brown et al., 2015; Castelle et al., 2015). In these efforts, the subsurface has emerged as a bountiful reservoir of undiscovered, deeply branching microbial lineages that may hold clues to the emergence and evolution of life on our planet (Kallmeyer et al., 2012; Colwell and D'Hondt, 2013). Many of the recently discovered candidate phyla are composed of microorganisms with streamlined genomes lacking some core metabolic pathways, which may be a factor contributing to the inability to obtain pure cultures of these organisms (Rinke et al., 2013; Becraft et al., 2015; Brown et al., 2015; Castelle et al., 2015). This apparent genomic reduction has given rise to hypotheses of genome-streamlining, parasitism, symbiotic lifestyles, and large-scale community metabolic inter-dependence (Giovannoni et al., 2014; Castelle et al., 2015; Anantharaman et al., 2016).

Our preliminary findings from several subsurface environments indicated that the 'Candidate phylum Spring Alpine Meadow' (SPAM) constitute an intriguing exception to genome streamlining in oligotrophic environments. The existence of this lineage was first suggested by several 16S rRNA gene sequences obtained in 2004 from an alpine soil from the Colorado Rocky mountains (Lipson, 2004). Subsequently, related 16S rRNA gene sequences were identified on all continents except for Antarctica in environments such as crop soils (Hansel et al., 2008; Chen et al., 2012; Figuerola et al., 2015), copper mine soil (Rodrigues et al., 2014), the subsurface oxic sediments of Hanford Formation at Pacific Northwest National Laboratory (PNNL) (Lin et al., 2012), subsurface groundwater from the Rifle site (Anantharaman et al., 2016), as well as volcanic mud and oil wells (unpublished) (Figure 1). 
82 different depths in Nevada, South Dakota and South Africa. We compare genomic data from

83 these individual cells to SPAM metagenome bins from Nevada groundwater, a Tabebuia

84 rhizosphere in Puerto Rico, and a prior study of the Rifle DOE Scientific Focus Area (SFA) in

85 Colorado (Anantharaman et al., 2016), where the first metagenome bins of these organisms were

86 obtained. This first global $16 \mathrm{~S}$ rRNA gene survey of SPAM suggests that they comprise a

87 monophyletic, phylum-level lineage that is most closely related to Nitrospirae. Different from the

88 Nitrospirae, SPAM genomes are consistently large, with high \%GC and the potential for a

89 mixotrophic metabolism, all packaged within small cells. SPAM cells are also characterized by

90 an unusually high genomic heterogeneity among individuals, with no environments identified to

91 date with near-clonal populations. The unique combination of large genomes encoding the ability

92 for a generalist metabolic strategy in oligotrophic environments, and contained within small

93 cells, is a rare observation among the recent explosion of candidate phyla characterization

94 (Castelle et al., 2015; Anantharaman et al., 2016; Hug et al., 2016b). High level of genetic

95 heterogeneity among SPAM individuals in studied samples is another intriguing feature that may

96 present certain challenges to their investigations.

97 Based on these observations, we propose renaming SPAM to the candidate phylum

98 "Candidatus Solagigasbacteria" (hereby referred to as Solagigasbacteria), with reference to

99 "sola" and "gigas" (Latin for "lone" and "giant"), encompassing the previously identified

100 Rokubacteria and the newly identified class in this study, the "Candidatus Infratellusbacteria",

101 with reference to "infra" and "tellus" (Latin for "below" and "Earth").

MATERIALS AND METHODS 

CO, Nevada, USA, named "Oasis Valley 2", hereafter referred to as "OV-2", on 14 December, $2014\left(36.96^{\circ} \mathrm{N},-116.72^{\circ} \mathrm{W}\right) . \mathrm{OV}-2$ is a PVC-cased hole, drilled in alluvial sand and gravel derived from tertiary volcanics to a depth of $36.5 \mathrm{~m}$ in 2011 . The well is screened (i.e. perforations were cut into the casing through which water can enter, but sand and other aquifer materials do not) over the interval from $9.1-27.4 \mathrm{~m}$. Samples OV-2 P1, P2 and P3 were collected after removal of one, three and ten well volumes at a pumping rate of $\sim 10,600 \mathrm{~L} / \mathrm{min}$. Microbial biomass was collected on $0.2 \mu \mathrm{m}$ polyethersulfone membrane filters (Millipore, Sterivex) from one, three, and five liters of samples at time points OV-2 P1, OV-2 P2, OV-2 P3, 114 respectively.

Discharge water samples were collected from the Crystal Spring, which is located adjacent to Death Valley, CA, USA on 13 December, $2014\left(36.42^{\circ} \mathrm{N},-116.72^{\circ} \mathrm{W}\right)$. Crystal

117 Spring is the largest spring of the largest Oasis of the Mojave Desert; Ash Meadows, Nye CO, $118 \mathrm{NV}$, USA. It is located within the discharge zone for a regional aquifer hosted within the highly 119 fractured Paleozoic carbonates in the Death Valley Regional Flow System (DVRFS) (Belcher et al., 2009). samples were also collected from a borehole at Finsch diamond mine at a depth of $857 \mathrm{mbls}$ in

126 South Africa on 11 November, $2012\left(-28.38^{\circ} \mathrm{S}, 23.45^{\circ} \mathrm{E}\right)$. 
129 milliliter aliquots were amended with 5\% glycerol and 1x TE buffer (all final concentrations), frozen on dry ice in the field and stored at $-80^{\circ} \mathrm{C}$ until further processing. For metagenomics, the DNA from OV-2 samples were extracted from microbial biomass collected on $0.2 \mu \mathrm{m}$ polyethersulfone membrane filters (Millipore, Sterivex) using the MO BIO PowerSoil DNA Isolation Kit (MO BIO Laboratories Inc., Carlsbad, CA) according to manufacturer's protocol. An additional freeze/thaw cycle was included after the addition of solution $\mathrm{C} 1$ and immediately prior to the ten-minute vortex step $\left(30 \mathrm{~min}\right.$ at $-80^{\circ} \mathrm{C}$ followed by $10 \mathrm{~min}$ at $\left.65^{\circ} \mathrm{C}\right)$. Additionally, a sample for metagenome sequencing was collected from a Tabebuia (T. heterophylla) rhizosphere in the serpentine area of Cabo Rojo Puerto Rico on 12 March, 2013. Three secondary roots from one tree, about $15-20 \mathrm{~cm}$ in length were collected, cut, stored in a $50 \mathrm{~mL}$ polyethylene centrifuge tube and transported on ice to the laboratory. The rhizosphere samples were obtained by washing the roots with $25 \mathrm{~mL} 1 \mathrm{X}$ PBS/Tween20 and shaken at $240 \mathrm{rpm}$ horizontally for 1 hour, and 141 frozen at $-80{ }^{\circ} \mathrm{C}$. The PBS/Tween20 solution with the rhizosphere was centrifuged at 9,000 $\mathrm{g}$ for

$14220 \mathrm{~min}$ at $4{ }^{\circ} \mathrm{C}$. Genomic DNA was extracted from the resulting pellet using the MoBio 143 PowerSoil DNA Isolation Kit with bead tubes (Carlsbad, CA) following Earth Microbiome 144 Project standard protocols (http://www.earthmicrobiome.org/protocols-and-standards/). Site 145 images and the physicochemical characteristics of these field samples are reported in 146 Supplemental Figure 2 and Supplemental Table 1.

\section{Single-cell genomics}

The generation, identification, sequencing and de novo assembly of single amplified

150 (scgc.bigelow.org). The cryopreserved samples were thawed, pre-screened through a $40 \mu \mathrm{m}$ 
153 using a BD InFlux Mariner flow cytometer equipped with a $488 \mathrm{~nm}$ laser and a $70 \mu \mathrm{m}$ nozzle 154 orifice (Becton Dickinson, formerly Cytopeia). The cytometer was triggered on side scatter, and 155 the "single-1 drop" mode was used for maximal sort purity. The sort gate was defined based on 156 particle green fluorescence (proxy to nucleic acid content), light side scatter (proxy to size), and 157 the ratio of green versus red fluorescence (for improved discrimination of cells from detrital 158 particles). Individual cells were deposited into 384-well plates containing $600 \mathrm{~nL}$ per well of 1x 159 TE buffer and stored at $-80^{\circ} \mathrm{C}$ until further processing. Of the 384 wells, 317 wells were 160 dedicated for single particles, 64 wells were used as negative controls (no droplet deposition), and 3 wells received 10 particles each to serve as positive controls. Index sort data was collected 162 using the BD FACS Sortware software. The DNA for each cell was amplified using WGA-X, as 163 previously described in Stepanauskas et al. (Stepanauskas et al., 2017). Cell diameters were determined using the FACS light forward scatter signal, which was calibrated against cells of 165 microscopy-characterized laboratory cultures (Stepanauskas et al., 2017). Illumina libraries were created, sequenced and de novo assembled as previously 167 described (Stepanauskas et al., 2017) This workflow was evaluated for assembly errors using 168 three bacterial benchmark cultures with diverse genome complexity and \%GC, indicating 60\% 169 average genome recovery, no non-target and undefined bases and the following, average 170 frequencies of misassemblies, indels and mismatches per $100 \mathrm{kbp}: 1.5,3.0$ and 5.0 (Stepanauskas 171 et al., 2017). checkM v1.0.6 (Parks et al., 2015) was used to calculate estimated the completeness 172 of assemblies of environmental SAGs. We did not co-assemble SAGs due to the high genomic 173 heterogeneity among individual cells. All SAGs were deposited in the Integrated Microbial 174 Genomes database at the Joint Genome Institute (accession numbers pending). 
177 General TimeReversible (GTR) Model, with Gamma distribution with invariable sites (G+I), and $17895 \%$ partial deletion for 1,000 replicate bootstraps. SAG assemblies were analyzed for protein 179 encoding regions using RAST (http://rast.nmpdr.org/) (Aziz et al., 2008), and genes (protein families) were annotated with Koala (KEGG) (http://www.kegg.jp/ghostkoala/) (Kanehisa et al., 2016) and InterProScan v5 (Jones et al., 2014). Average nucleotide identity (ANI) and average amino acid identity (AAI) of reciprocal hits were calculated using the online tools at the Kostas Lab website Environmental Microbial Genomics Laboratory (http://enveomics.ce.gatech.edu/aai/) (Goris et al., 2007; Rodriguez R and Konstantinidis, 2014). Synteny plots were produced using the Joint Genome Institute Integrated Microbial Genomes (IMG) system (https://img.jgi.doe.gov/) (Markowitz et al., 2014). Phage genes and transposases were identified as in Labonte et al. (Labonte et al., 2015b).

\section{Metagenomic sequencing and analysis}

For OV-2 samples, $1 \mathrm{ng}$ of DNA was fragmented and adapter ligated using the Nextera compatible adapters (IDT, Inc) using the KAPA-Illumina library creation kit (KAPA 
201 and removing reads containing unique kmers. The resulting filtered and error-corrected reads

202 were assembled for each sample separately using SPAdes v.3.9.0 without error correction with

203 kmers 27, 47, 67, 87, 107 (Bankevich et al., 2012). Reads were mapped to the assemblies using

204 Burrows-Wheel Aligner (BWA) v0.7.15 (Li and Durbin, 2010) and binned based on abundance

205 patterns and kmer composition using Metabat v0.32.4 with minimum contig length of $3 \mathrm{~kb}$ and

206 superspecific probability option (Kang et al., 2015). Differential coverage could not be utilized

207 as there was little overlap between the 3 OV-2 samples (i.e. less than $10 \%$ of the reads from P1

208 and P2 could be mapped to P3, and vice versa). The bins corresponding to Solagigasbacteria

209 were identified based on the presence of Solagigasbacteria 16S rRNA on contigs longer than 20

$210 \mathrm{~kb}$, as well as best BLAST hits to Solagigasbacteria SAG assemblies. Additional

211 Solagigasbacteria metagenome bins were identified by BLASTing annotated gene regions of

212 SAGs against metagenome assemblies, and bins with $\geq 200$ hits with $\leq 1 \mathrm{e}-50$ evalue score were

213 further analyzed with checkM v1.0.6. Bins with excessive contamination were ignored.

214 Metagenome assemblies are deposited in the Integrated Microbial Genomes database at the Joint

215 Genome Institute (3300009626, 3300009691, 3300009444 and 3300003659).

Recruitment of metagenome reads to single-amplified genomes (SAGs) was determined

217 using in-house software and Burrows-Wheel Aligner (BWA) v0.7.15 (Li and Durbin, 2010) to

218 map sequence reads to Solagigasbacteria SAG contigs that met the criteria of 100 bps overlap at

$219 \geq 90 \%$ nucleotide identity. The relative abundance of SAG relatives was determined as the

220 fraction of metagenome reads mapping per megabase of a reference genome.

221

222 RESULTS AND DISCUSSION

223

16S rRNA gene phylogeny and biogeography 
We used full-length $16 \mathrm{~S}$ rRNA gene sequences of Solagigasbacteria SAGs as queries in

225

226

227

228

229

230

231

232

233

234

235

236

237

238

239

240

241

242

243

244

245

246

247

BLASTn searches for related sequences in the NCBI nucleotide database that yielded 91 unique sequences $\geq 85 \%$ nucleotide identity over $\geq 600$ bps. A phylogenetic analysis of these sequences suggested that Solagigasbacteria form a strongly bootstrap-supported, monophyletic lineage (Figure 1). Nitrospirae was the most closely related phylum, sharing $79-83 \%$ 16S rRNA gene sequence identity with Solagigasbacteria. Some Solagigasbacteria 16S rRNA gene sequences were misclassified as Nitrospirae in public databases (green arrows in Figure 1, also see Supplemental Figure 3). Given that the Solagigasbacteria are a bootstrap-supported, monophyletic clade, contain unifying genomic features (e.g. GC content), and fall below the median phylum-level 16S rRNA gene similarity threshold of $83.68 \%$ (range 81.6-85.93\%) (Yarza et al., 2014), we propose that Solagigasbacteria is a unique phylum-level lineage. Phylogenies based on ribosomal preotein sequences (Hug et al., 2016a) (Anantharaman et al., 2016) agree with this phylogenetic placement and phylum demarcation. A superphylum may be formed by Solagigasbacteria, Aminicenantes, Acidobacteria, and the Candidate phylum NC10, but further phylogenomic analyses are needed to confirm this hypothesis.

The Solagigasbacteria 16S rRNA gene sequences form two deeply branching sublineages that diverge from each other by $\sim 12-15 \%$, i.e. at an operationally-defined class level (Figure 1; Supplemental Table 2) (Hugenholtz et al., 1998; Yarza et al., 2014). Apart from SAGs and PCR-derived sequences, one of the sub-lineages also included 16S rRNA genes from metagenome bins obtained from the Puerto Rican Tabebuia rhizosphere (light blue square in Figure 1) and from a previously published bin from the Rifle site, Colorado (orange square in Figure 1) (Anantharaman et al., 2016). The latter study named this lineage candidate phylum Rokubacteria, and we propose retaining this name for a class within Solagigasbacteria. Rokubacteria encompassed the majority of Solagigasbacteria sequences originating from both 
248 soils and shallow terrestrial subsurface environments. The majority of Rokubacteria SAGs from

249 OV-2 fell into a subclade comprised exclusively of 16S rRNA gene sequences from subsurface

250 sites. The second class-level lineage included a smaller set of sequences that originate

251 exclusively from terrestrial subsurface sites. We propose naming this candidate class

252 "Candidatus Infratellusbacteria" (hereby referred to as Infratellusbacteria), in order to reflect the

253 predominant environment in which these microorganisms have been detected so far.

The sources of samples from which Solagigasbacteria 16S sequences were retrieved (25

255 in total; including 19 previously sampled sites (Figure 1), suggest a cosmopolitan distribution in

256 soils and terrestrial subsurface, with no evidence so far for presence in marine environments.

257 Interestingly, Solagigasbacteria were low in abundance at almost every site where they were

258 identified in this and prior studies (Lin et al., 2012; Figuerola et al., 2015), and often were

259 represented by a single $16 \mathrm{~S}$ rRNA gene sequence. An alternative analysis of Solagigasbacteria

260 abundance in our study sites, by performing metagenome fragment recruitment on SAGs as

261 references, provided further evidence that Solagigasbacteria comprised $\sim 1 \%$ of the microbial

262 community in OV-2 (Supplemental Figure 4), similar to other samples (Lipson, 2004; Hansel et

263 al., 2008; Chen et al., 2012; Lin et al., 2012; Rodrigues et al., 2014; Figuerola et al., 2015). A

264 recent study identified Rokubacteria to constitute $\sim 10 \%$ of the microbial community in a grass

265 root zone in the Angelo Coast Range Reserve, California, making it the most Solagigasbacteria-

266 rich environment to date (Butterfield et al., 2016), though no 16S rRNA sequences were

267 identified in the metagenome bins.

\section{General genome features}

The SAGs obtained from SURF, Finsch, OV-2 and Crystal Spring sites contained

270 phylogenetically diverse representatives of both Solagigasbacteria classes, enabling us to explore

271 their genomic content, metabolic potential and evolutionary histories. De novo genome 
272 assemblies of the 19 SAGs ranged from 0.05 to $2.86 \mathrm{Mbps}$ (Table 1). The estimated

273 Solagigasbacteria genome completeness ranged between $1-40 \%$ (average of $18.2 \%$ ). This is

274 significantly lower than the genome recovery from other SAGs using the same techniques in 275 earlier studies, which averaged at around $50 \%$ (Rinke et al., 2013; Swan et al., 2013; Kashtan et 276 al., 2014). Based on the presence of conserved single copy genes in the most complete SAG 277 assemblies, we estimate that Solagigasbacteria complete genomes are $6-8$ Mbps in length 278 (average 6.8 Mbps; Table 1 and Supplemental Figure 5), which is slightly larger than estimates 279 obtained from metagenome bins at the Rifle site (4 - 6 Mbps; Supplemental Table 3) 280 (Anantharaman et al., 2016) and the Puerto Rican soil (Table 1). Genome size predictions for 281 smaller SAGs and contaminated metagenome bins are variable, though the more complete SAGs 282 and metagenome bins converge on the average genome size reported above (Supplemental 283 Figure 5). A relatively large fraction, between $8-17 \%$ of the Solagigasbacteria genomes, 284 consists of nucleotides predicted to be non-coding. With a few intriguing exceptions (Sekiguchi 285 et al., 2015), these features present a stark contrast to the predominantly small and streamlined 286 genomes of most recently described bacterial and archaeal candidate phyla from diverse surface 287 and subsurface environments, including the abundant and diverse candidate superphylum 288 Patescibacteria (Rinke et al., 2013), which was later proposed to constitute an even larger 289 evolutionary unit, the Candidate Phyla Radiation (CPR) (Rinke et al., 2013; Brown et al., 2015; 290 Castelle et al., 2015; Anantharaman et al., 2016). 295 phylum to Solagigasbacteria, Nitrospirae, has a more variable GC content, ranging from $34 \%$ 

Petrov, 2010). Factors determining high \%GC remain controversial, with some studies suggesting the importance of temperature and solar radiation as selective variables (Foerstner et al., 2005; Hildebrand et al., 2010), while other reports refute these findings (Lassalle et al., 2015; within lineages (Lassalle et al., 2015; Reichenberger et al., 2015), other studies show large GC variation among lineages that were thought to be exclusively high in GC, such as the Actinobacteria phylum (Ghai et al., 2012; Swan et al., 2013) and the Roseobacter clade of Alphaproteobacteria (Swan et al., 2013; Zhang et al., 2016). The high \%GC of Solagigasbacteria contrasts low \%GC in most of the major, uncultured branches of Bacteria and Archaea explored with single-cell genomics (Rinke et al., 2013) and metagenome binning (Anantharaman et al.,

312 processes are involved in the emergence and maintenance of high $\% \mathrm{GC}$, and to what extent has 313 the discovery of novel microbial lineages with high \%GC been hampered by biases in DNA 314 amplification (Stepanauskas et al., 2017) and sequencing techniques (Chen et al., 2013).

317 identity (AAI) was 46.2\% (range from 34.6 - 64.2\%; Figure 3), demonstrating high cell-to-cell 318 genome divergence. Interestingly, SAGs originating from the OV-2 sample shared roughly the 
320 divergent Solagigasbacteria SAGs were obtained from the same OV-2 site (Figure 1), both

321 within and between class-level lineages. Genomes were mostly non-syntenic on larger scales.

322 However, many shared proteins of related function were located in small islands of synteny in

323 the six least fragmented SAG assemblies (Supplemental Figure 6). Causes for the unusually

324 variable genome content among cells in each study site remain unclear. Dispersal of dormant

325 cells to the sampling sites from a multitude of evolutionarily distant populations is one plausible

326 explanation. An alternative explanation may be the accumulation of point mutations, gene

327 acquisitions, gene loss and genome rearrangements at a rate that outpaces cell division. The latter

328 possibility is highly speculative and contradicts conventional models of microbial evolution, but

329 should be viewed in the context of bacterial generation times potentially ranging in hundreds and

330 even thousands of years in some low-energy, subsurface environments (Labonte et al., 2015a).

Solagigasbacteria genomes contain numerous transposases and integrases $(4-60$ per

SAG assembly; Supplemental Table 4). Genes of potential viral origin and CRISPR regions were

contained phage-like genes were never predicted to be entirely viral, indicating integration into

prophages in Firmicutes in the deep subsurface of the Witwatersrand Basin (Labonte et al., 2015a) and indicate a potentially important role of viruses as vectors of horizontal gene transfer in low-energy, subsurface environments.

\section{Predicted phenotype and energy production}

against a series of benchmark cultures, to estimate approximate diameters of the cells from 
$3440.15-0.20 \mu \mathrm{m}$ diameter reported for some of the CPR cells (Luef et al., 2015), and the $\sim 0.2-$

$3450.3 \mu \mathrm{m}$ average diameter of the most abundant marine bacterioplankton lineage Pelagibacter

346 (Giovannoni et al., 2005; Giovannoni et al., 2014), it is approaching the theoretical lower limit

347 for cell sizes (NRC., 1999). Such small cells, including the CPR, Pelagibacter, Mycoplasma,

348 ultrasmall Actinomycetes, and Prochlorococcus, have extremely small, streamlined genomes that range between 0.8 - 2.5 Mbps (Biller et al., 2014; Nakai et al., 2016; Parrott et al., 2016). In the case of Solagigasbacteria, the presence of large genomes in small cells may imply extensive DNA packaging or dormancy. In partial support of this hypothesis, a variety of DNA packaging and super-coiling proteins where annotated in the Solagigasbacteria SAGs and metagenome bins (Supplementary Table 5). Further experimental work is required to confirm these predictions. organisms, including the majority of genes involved in the production and transport of lipids across the cytoplasmic membrane for outer membrane and LPS assembly (Sutcliffe, 2010)

357 (Supplemental Figure 1 and Supplemental Table 5), which is consistent with their phylogenetic 358 affiliation with the Gram-negative Nitrospirae. We identified multiple genes involved in 359 twitching motility in 11 Rokubacteria SAGs, 4 Infratellusbacteria SAGs, and both metagenome 360 bins, possibly indicating a conserved mechanism of pili motility in the Solagigasbacteria 361 (Supplemental Table 5). We also identified genes in 3 Rokubacteria SAGs and Infratellusbacteria OV-2 bin 8 that are predicted to encode flagella structural proteins, while 363 propeller filament genes were absent in all SAG assemblies and metagenome bins (Supplemental 364 Figure 1). While OV-2 bin 8 contained genes involved in flagella assembly, Infratellusbacteria 365 SAGs lacked genes required for the assembly of flagella, though gene absence could be due to fewer and less complete SAG assemblies. Furthermore, putative genes were identified in the 367 majority of assemblies for methyl-accepting chemotaxis proteins, two-component sensor kinases, 
368 ATP motor proteins, and sensor proteins for nitrogen, oxygen, zinc/lead, and acetoacetate,

369 indicating that Solagigasbacteria can respond to a broad range of chemical stimuli.

370 Solagigasbacteria contain multiple carbon transport proteins, including those specializing in

371 lipids, peptides and sugars, and carbon degradation and election transport pathways for aerobic

372 respiration (Supplemental Figure 1). Rokubacteria SAGs also contain genes involved in nitrogen

373 respiration, including nitrite oxidoreductases, which are universally conserved in the Nitrospirae

374 lineage (Supplemental Figure 7). See Supplemental Section I for detailed metabolic predictions.

375

376

377

378

379

380

381

382

383

384

385

386

387

388

389

390

\section{Comparison of Solagigasbacteria assemblies from single cells and metagenomes}

The availability of several partial genomes of Solagigasbacteria from single cells and metagenomes from this and prior studies (Table 1 and Supplemental Table 3) offered an opportunity to compare the type and quality of information that can be extracted using these two approaches. Genome completeness is one important quality metric of de novo assemblies. The ratio of the number of single copy marker genes that are found versus expected in an assembly is the most commonly use proxy for genome completeness and is implemented in popular computational tools, such as checkM (Parks et al., 2015). In our study, checkM-based estimates of assembly completeness of Solagigasbacteria SAGs and metagenome bins ranged between 1$40 \%$ and $14-100 \%$, respectively, suggesting that individual SAG assemblies tended to be less complete than metagenome bins (Table 1). However, the higher average completeness of metagenome bins came with important caveats: high estimated contamination in five out of eight bins (Table 1), absence of rRNA genes in six out of eight bins, and the lack of knowledge of the number and genetic diversity of cells that contributed genomic sequences to each bin. These caveats may limit the interpretability of metagenome bins in the context of microbial ecology and evolution and outweigh the benefits of their higher estimated completeness. 

agreement with the recent benchmarking effort employing $>1,000$ previously sequenced strains of microorganisms and mobile genetic elements, which found that the performance of metagenome assembly and binning is impaired by the presence of related strains in a sample (Sczyrba, 2017).

The checkM estimates of contamination are based on the phylogenetic placement of the assembly's single copy marker genes against a built-in database (Parks et al., 2015), which lacks many uncultured lineages, including Solagigasbacteria. To the best of our knowledge, the ability of checkM to detect contamination that originates from lineages that are absent from its database

403 has never been evaluated. To address this question, we created pairwise combinations of assemblies of each Infratellusbacteria SAG with each Rokubacteria SAG. The checkM-estimated 405 contamination in these combined assemblies was significantly smaller than the real, cross-class contamination (Figure 5), suggesting that checkM may fail detecting contamination from

407 lineages not represented in the checkM database. Strikingly, the majority of our artificial combinations of SAGs from different phylogenetic classes would be considered 'high quality' genomes according to recently proposed genome standards for SAGs and metagenome bins

410 (Bowers, 2017). In order to assess whether similar, cross-class contamination may be affecting

411 our metagenome bins, we analyzed AAI among Solagigasbacteria SAGs and the only OV2 412 metagenome bin that contained a rRNA gene (bin8). While the rRNA gene placed this bin firmly 413 among the Infratellusbacteria (Figure 1), its AAI suggested affiliation with Rokubacteria (Figure 414 3). Furthermore, the best BLAST hits to bin8 genes consisted of SAGs from both class-level 
415 lineages, including multiple near full-length alignments at >95\% nucleotide identity with

416 Rokubacteria SAGs (Supplemental Table 6). This indicates that the checkM-based estimate of

$4172 \%$ contamination for this bin may be a major underestimate. These findings imply that

418 improvements are urgently needed in the quality control of genome assemblies originating from

419 uncultured microbial groups and in the validation of the performance of QC software.

420 The comparison of SAGs and metagenome bins demonstrates that the two approaches

421 provide two fundamentally different types of data and should be interpreted accordingly. While

422 SAG assemblies represent fragments of discrete genomes from individual cells, the metagenome

423 bins are fragments of consensus sequences derived from a multitude of genetically non-identical

424 organisms. The consistency of certain general features between Solagigasbacteria SAGs and bins

425 (e.g. high \%GC, large estimated genome size, and many shared metabolic pathways) suggests

426 that metagenome bins provide useful consensus information about this candidate phylum (Figure

4272 and Supplemental Figure 1). However, consensus sequences appear to mask extensive genetic

428 diversity among Solagigasbacteria cells in the studied environments. On a more fundamental

429 level, metagenome assembly and binning relies on the assumption that microbial communities

430 are composed of near-clonal populations. An increasing body of evidence shows that this

431 assumption is not valid in many microorganismal lineages and environments, with genomic

432 rearrangements and horizontal gene transfer being more prevalent than previously thought

433 (Ochman et al., 2000; Feldgarden et al., 2003; Shapiro, 2010; Kashtan et al., 2014; Labonte et

434 al., 2015a). By recovering data from the most fundamental units of biological organization,

435 single-cell genomics does not rely on the assumption of clonality, offers an opportunity to

436 improve our understanding of microbial microevolutionary processes (Garrity and Lyons, 2003;

437 Engel et al., 2014; Kashtan et al., 2014), and helps calibrating the performance and interpretation 
438 of metagenomics tools when working with complex, natural microbial assemblages (Becraft et 439 al., 2015).

440

441

442

443

444

445

446

447

448

449

450

451

452

453

454

455

456

457

458

459

460

CONCLUDING REMARKS

Recent discoveries of many novel phyla and superphyla of microorganisms are revolutionizing our understanding of the genealogy and current diversity of life. Here, a focused analysis of the single cell genomic and metagenome sequences of Solagigasbacteria (formerly known as SPAM) suggests that they constitute a monophyletic, phylum-level lineage that is most closely related to Nitrospirae among the currently described phyla. Large genomes, high \%GC, and a global presence at low abundance in soils and terrestrial subsurface environments appear to be general features of this candidate phylum. Solagigasbacteria genomes predict didermy, mixotrophy, motility, and versatile DNA packaging mechanisms. It is plausible that the latter feature interferes with gDNA amplification, in part explaining the difficulty of recovering high quality genomes from Solagigasbacteria single cells. Furthermore, large cell-to-cell genomic heterogenetity and low relative abundance in most environments studied to date may be among the factors contributing to their limited recovery in metagenome bins. Our analysis also demonstrates major differences in the quality of genomic data obtained from SAGs and metagenome bins: While assemblies with greatest estimated genome recovery where obtained by metagenome binning, SAGs delivered contamination-free data from discrete biological units, making them easier to interpret and revealing significant genomic diversity within this candidate phylum, including a split into two class-level lineages.

\section{ACKNOWLEDGEMENTS}


Olukayode Kuloyo, Borja Linage, Sarah Hendrickson, Cara Magnabosco, Melody Lindsay,

Department of Energy Joint Genome Institute is supported by the Office of Science of the U.S.

there are no conflicts of interest.

\section{REFERENCES}

Anantharaman, K., Brown, C.T., Hug, L.A., Sharon, I., Castelle, C.J., Probst, A.J. et al. (2016) Thousands of microbial genomes shed light on interconnected biogeochemical processes in an aquifer system. Nat Commun 7: 13219.

Aziz, R.K., Bartels, D., Best, A.A., DeJongh, M., Disz, T., Edwards, R.A. et al. (2008) The RAST Server: Rapid Annotations using Subsystems Technology. BMC Genomics 9: 75.

Bankevich, A., Nurk, S., Antipov, D., Gurevich, A.A., Dvorkin, M., Kulikov, A.S. et al. (2012) SPAdes: a new genome assembly algorithm and its applications to single-cell sequencing. J Comput Biol 19: 455-477.

Becraft, E.D., Dodsworth, J.A., Murugapiran, S.K., Ohlsson, J.I., Briggs, B.R., Kanbar, J. et al. (2015) Single-Cell-Genomics-Facilitated Read Binning of Candidate Phylum EM19 Genomes from Geothermal Spring Metagenomes. Appl Environ Microbiol 82: 992-1003.

Belcher, C.M., Finch, P., Collinson, M.E., Scott, A.C., and Grassineau, N.V. (2009) Geochemical evidence for combustion of hydrocarbons during the K-T impact event. Proc Natl Acad Sci USA 106: 4112-4117.

Biller, S.J., Berube, P.M., Berta-Thompson, J.W., Kelly, L., Roggensack, S.E., Awad, L. et al. (2014) Genomes of diverse isolates of the marine cyanobacterium Prochlorococcus. Sci Data 1: 140034.

Bolger, A.M., Lohse, M., and Usadel, B. (2014) Trimmomatic: A flexible trimmer for Illumina sequence data. Bioinformatics 30: 2114-2120.

Bowers, R., Nikos C. Kyrpides, Ramunas Stepanauskas, Miranda Harmon-Smith, Frederik Schulz, Devin Doud, T.B.K. Reddy, Jessica Jarett, Adam R. Rivers, Emiley A. Eloe-Fadrosh, Susannah G. Tringe, Natalia Ivanova, Alex Copeland, Alicia Clum, Eric D. Becraft, Rex R. Malmstrom, Bruce Birren, Lynn Schriml, Mircea Podar, Peer Bork, George M Weinstock, Jillian 
F Banfield, George M. Garrity, Philip Hugenholtz, Donovan H. Parks, Gene W. Tyson, Christian Rinke, Jeremy A. Dodsworth, Shibu Yooseph, Granger Sutton, Pelin Yilmaz, Frank Oliver Glöckner, Folker Meyer, Jack A. Gilbert, William C. Nelson, Steven J. Hallam, Sean P. Jungbluth, Thijs J. G. Ettema, Scott Tighe, Konstantinos T Konstantinidis, Alla Lapidus, WenTso Liu, Brett J. Baker, Thomas Rattei, Jonathan A. Eisen, Brian Hedlund, Katherine D. McMahon, Noah Fierer, Rob Knight, Rob Finn, Ilene Karsch-Mizrachi, A.M. Eren and Tanja Woyke (2017) Minimum information about a single amplified genome (MISAG) and a metagenome-assembled genome (MIMAG) of bacteria and archaea. Nature Biotechnology 35: 725-731.

Brown, C.T., Hug, L.A., Thomas, B.C., Sharon, I., Castelle, C.J., Singh, A. et al. (2015) Unusual biology across a group comprising more than $15 \%$ of domain Bacteria. Nature 523: 208-211.

Butterfield, C.N., Li, Z., Andeer, P.F., Spaulding, S., Thomas, B.C., Singh, A. et al. (2016) Proteogenomic analyses indicate bacterial methylotrophy and archaeal heterotrophy are prevalent below the grass root zone. PeerJ 4: e2687.

Castelle, C.J., Wrighton, K.C., Thomas, B.C., Hug, L.A., Brown, C.T., Wilkins, M.J. et al. (2015) Genomic expansion of domain archaea highlights roles for organisms from new phyla in anaerobic carbon cycling. Curr Biol 25: 690-701.

Chen, X., Su, Y., He, X., Wei, Y., Wei, W., and Wu, J. (2012) Soil bacterial community composition and diversity respond to cultivation in Karst ecosystems. World $\mathrm{J}$ Microbiol Biotechnol 28: 205-213.

Chen, Y.C., Liu, T., Yu, C.H., Chiang, T.Y., and Hwang, C.C. (2013) Effects of GC bias in next-generation-sequencing data on de novo genome assembly. PLoS One 8: e62856.

Colwell, F.S., and D'Hondt, S. (2013) Nature and Extent of the Deep Biosphere. Reviews in Mineralogy and Geochemistry 75: 547-574.

Engel, P., Stepanauskas, R., and Moran, N.A. (2014) Hidden diversity in honey bee gut symbionts detected by single-cell genomics. PLoS Genet 10: e1004596.

Feldgarden, M., Byrd, N., and Cohan, F.M. (2003) Gradual evolution in bacteria: evidence from Bacillus systematics. Microbiology 149: 3565-3573.

Figuerola, E.L., Guerrero, L.D., Turkowsky, D., Wall, L.G., and Erijman, L. (2015) Crop monoculture rather than agriculture reduces the spatial turnover of soil bacterial communities at a regional scale. Environ Microbiol 17: 678-688.

Foerstner, K.U., von Mering, C., Hooper, S.D., and Bork, P. (2005) Environments shape the nucleotide composition of genomes. EMBO Rep 6: 1208-1213.

Garrity, G.M., and Lyons, C. (2003) Future-proofing biological nomenclature. OMICS 7: 3133.

Ghai, R., McMahon, K.D., and Rodriguez-Valera, F. (2012) Breaking a paradigm: cosmopolitan and abundant freshwater actinobacteria are low GC. Environ Microbiol Rep 4: 2935 .

Giovannoni, S.J., Cameron Thrash, J., and Temperton, B. (2014) Implications of streamlining theory for microbial ecology. ISME J 8: 1553-1565.

Giovannoni, S.J., Tripp, H.J., Givan, S., Podar, M., Vergin, K.L., Baptista, D. et al. (2005) Genome streamlining in a cosmopolitan oceanic bacterium. Science 309: 1242-1245.

Goris, J., Konstantinidis, K.T., Klappenbach, J.A., Coenye, T., Vandamme, P., and Tiedje, J.M. (2007) DNA-DNA hybridization values and their relationship to whole-genome sequence similarities. International Journal Of Systematic and Evolutionary Microbiology 57: 81-91. 
Hansel, C.M., Fendorf, S., Jardine, P.M., and Francis, C.A. (2008) Changes in bacterial and archaeal community structure and functional diversity along a geochemically variable soil profile. Appl Environ Microbiol 74: 1620-1633.

Hershberg, R., and Petrov, D.A. (2010) Evidence that mutation is universally biased towards AT in bacteria. PLoS Genet 6: e1001115.

Hildebrand, F., Meyer, A., and Eyre-Walker, A. (2010) Evidence of selection upon genomic GC-content in bacteria. PLoS Genet 6: e1001107.

Hug, L.A., Thomas, B.C., Sharon, I., Brown, C.T., Sharma, R., Hettich, R.L. et al. (2016a) Critical biogeochemical functions in the subsurface are associated with bacteria from new phyla and little studied lineages. Environ Microbiol 18: 159-173.

Hug, L.A., Baker, B.J., Anantharaman, K., Brown, C.T., Probst, A.J., Castelle, C.J. et al. (2016b) A new view of the tree of life. Nature Microbiology: 16048.

Hugenholtz, P., Pitulle, C., Hershberger, K.L., and Pace, N.R. (1998) Novel division level bacterial diversity in a Yellowstone hot spring. J Bacteriol 180: 366-376.

Jones, P., Binns, D., Chang, H.Y., Fraser, M., Li, W., McAnulla, C. et al. (2014) InterProScan 5: genome-scale protein function classification. Bioinformatics 30: 1236-1240.

Kallmeyer, J., Pockalny, R., Adhikari, R.R., Smith, D.C., and D'Hondt, S. (2012) Global distribution of microbial abundance and biomass in subseafloor sediment. Proc Natl Acad Sci USA 109: 16213-16216.

Kanehisa, M., Sato, Y., and Morishima, K. (2016) BlastKOALA and GhostKOALA: KEGG Tools for Functional Characterization of Genome and Metagenome Sequences. J Mol Biol 428: 726-731.

Kang, D.D., Froula, J., Egan, R., and Wang, Z. (2015) MetaBAT, an efficient tool for accurately reconstructing single genomes from complex microbial communities. PeerJ 3: e1165.

Kashtan, N., Roggensack, S.E., Rodrigue, S., Thompson, J.W., Biller, S.J., Coe, A. et al. (2014) Single-cell genomics reveals hundreds of coexisting subpopulations in wild Prochlorococcus. Science 344: 416-420.

Labonte, J.M., Field, E.K., Lau, M., Chivian, D., Van Heerden, E., Wommack, K.E. et al. (2015a) Single cell genomics indicates horizontal gene transfer and viral infections in a deep subsurface Firmicutes population. Front Microbiol 6: 349.

Labonte, J.M., Swan, B.K., Poulos, B., Luo, H., Koren, S., Hallam, S.J. et al. (2015b) Singlecell genomics-based analysis of virus-host interactions in marine surface bacterioplankton. ISME J 9: 2386-2399.

Lassalle, F., Perian, S., Bataillon, T., Nesme, X., Duret, L., and Daubin, V. (2015) GCContent evolution in bacterial genomes: the biased gene conversion hypothesis expands. PLoS Genet 11: e1004941.

Li, H. (2015) BFC: correcting Illumina sequencing errors. Bioinformatics 31: 2885-2887.

Li, H., and Durbin, R. (2010) Fast and accurate long-read alignment with Burrows-Wheeler transform. Bioinformatics 26: 589-595.

Li, J., Zhou, J., Wu, Y., Yang, S., and Tian, D. (2015) GC-Content of Synonymous Codons Profoundly Influences Amino Acid Usage. G3 (Bethesda) 5: 2027-2036.

Lin, X., Kennedy, D., Fredrickson, J., Bjornstad, B., and Konopka, A. (2012) Vertical stratification of subsurface microbial community composition across geological formations at the Hanford Site. Environ Microbiol 14: 414-425.

Lipson, D.A., Schmidt, S.K. (2004) Seasonal Changes in an Alpine Soil Bacterial Community in the Colorado Rocky Mountains. Applied and Environmental Microbiology 70: 2867-2879. 
Luef, B., Frischkorn, K.R., Wrighton, K.C., Holman, H.Y., Birarda, G., Thomas, B.C. et al. (2015) Diverse uncultivated ultra-small bacterial cells in groundwater. Nat Commun 6: 6372.

Markowitz, V.M., Chen, I.M., Palaniappan, K., Chu, K., Szeto, E., Pillay, M. et al. (2014) IMG 4 version of the integrated microbial genomes comparative analysis system. Nucleic Acids Res 42: D560-567.

Moriya, Y., Itoh, M., Okuda, S., Yoshizawa, A.C., and Kanehisa, M. (2007) KAAS: an automatic genome annotation and pathway reconstruction server. Nucleic Acids Research 35: W182-W185.

Nakai, R., Fujisawa, T., Nakamura, Y., Nishide, H., Uchiyama, I., Baba, T. et al. (2016) Complete Genome Sequence of Aurantimicrobium minutum Type Strain KNCT, a Planktonic Ultramicrobacterium Isolated from River Water. Genome Announc 4.

NRC., S.G.f.t.W.o.s.l.o.v.s.M. (1999) Size Limits of Very Small Microorgansisms. In: National Academy Press.

Ochman, H., Lawrence, J.G., and Groisman, E.A. (2000) Lateral gene transfer and the nature of bacterial innovation. Nature 405: 299-304.

Parks, D.H., Imelfort, M., Skennerton, C.T., Hugenholtz, P., and Tyson, G.W. (2015) CheckM: assessing the quality of microbial genomes recovered from isolates, single cells, and metagenomes. Genome Res 25: 1043-1055.

Parrott, G.L., Kinjo, T., and Fujita, J. (2016) A Compendium for Mycoplasma pneumoniae. Front Microbiol 7: 513.

Pruesse, E., Peplies, J., and Glockner, F.O. (2012) SINA: Accurate high-throughput multiple sequence alignment of ribosomal RNA genes. Bioinformatics 28: 1823-1829.

Reichenberger, E.R., Rosen, G., Hershberg, U., and Hershberg, R. (2015) Prokaryotic nucleotide composition is shaped by both phylogeny and the environment. Genome Biol Evol 7: 1380-1389.

Rinke, C., Schwientek, P., Sczyrba, A., Ivanova, N.N., Anderson, I.J., Cheng, J.-F. et al. (2013) Insights into the phylogeny and coding potential of microbial dark matter. Nature 499: 431-437.

Rodrigues, V.D., Torres, T.T., and Ottoboni, L.M. (2014) Bacterial diversity assessment in soil of an active Brazilian copper mine using high-throughput sequencing of 16S rDNA amplicons. Antonie Van Leeuwenhoek 106: 879-890.

Rodriguez R and Konstantinidis, K. (2014) Bypassing Cultivation To Identify Bacterial Species. In Microbe.

Sczyrba, A. (2017) Critical Assessment of Metagenome Interpretation - a benchmark of computational metagenomics software. bioRxiv.

Sekiguchi, Y., Ohashi, A., Parks, D.H., Yamauchi, T., Tyson, G.W., and Hugenholtz, P. (2015) First genomic insights into members of a candidate bacterial phylum responsible for wastewater bulking. PeerJ 3: e740.

Shapiro, J.A. (2010) Mobile DNA and evolution in the 21st century. Mob DNA 1: 4.

Sorokin, D.Y., Lucker, S., Vejmelkova, D., Kostrikina, N.A., Kleerebezem, R., Rijpstra, W.I. et al. (2012) Nitrification expanded: discovery, physiology and genomics of a nitrite-oxidizing bacterium from the phylum Chloroflexi. ISME J 6: 2245-2256.

Stepanauskas, R., Fergusson, E.A., Brown, J., Poulton, N.J., Tupper, B., Labonte, J.M. et al. (2017) Improved genome recovery and integrated cell-size analyses of individual uncultured microbial cells and viral particles. Nat Commun 8: 84 .

Sutcliffe, I.C. (2010) A phylum level perspective on bacterial cell envelope architecture. Trends in Microbiology 18: 464-470. 
635

636

637

638

639

640

641

642

643

644

645

646

647

648

649

650

651

652

653

654

655

656

657

658

659

660

661

Swan, B.K., Tupper, B., Sczyrba, A., Lauro, F.M., Martinez-Garcia, M., Gonzalez, J.M. et al. (2013) Prevalent genome streamlining and latitudinal divergence of planktonic bacteria in the surface ocean. Proc Natl Acad Sci USA 110: 11463-11468.

Tamura, K., Stecher, G., Peterson, D., Filipski, A., and Kumar, S. (2013) MEGA6: Molecular Evolutionary Genetics Analysis Version 6.0. Molecular Biology and Evolution 30: 2725-2729.

Yarza, P., Yilmaz, P., Pruesse, E., Glöckner, F.O., Ludwig, W., Schleifer, K.-H. et al. (2014) Uniting the classification of cultured and uncultured bacteria and archaea using 16S rRNA gene sequences. Nature Reviews Microbiology 12: 635-645.

Yoon, H.S., Price, D.C., Stepanauskas, R., Rajah, V.D., Sieracki, M.E., Wilson, W.H. et al. (2011) Single-cell genomics reveals organismal interactions in uncultivated marine protists. Science 332: 714-717.

Zhang, Y., Sun, Y., Jiao, N., Stepanauskas, R., and Luo, H. (2016) Ecological Genomics of the Uncultivated Marine Roseobacter Lineage CHAB-I-5. Appl Environ Microbiol 82: 21002111.

(1)

5

56

\section{7}




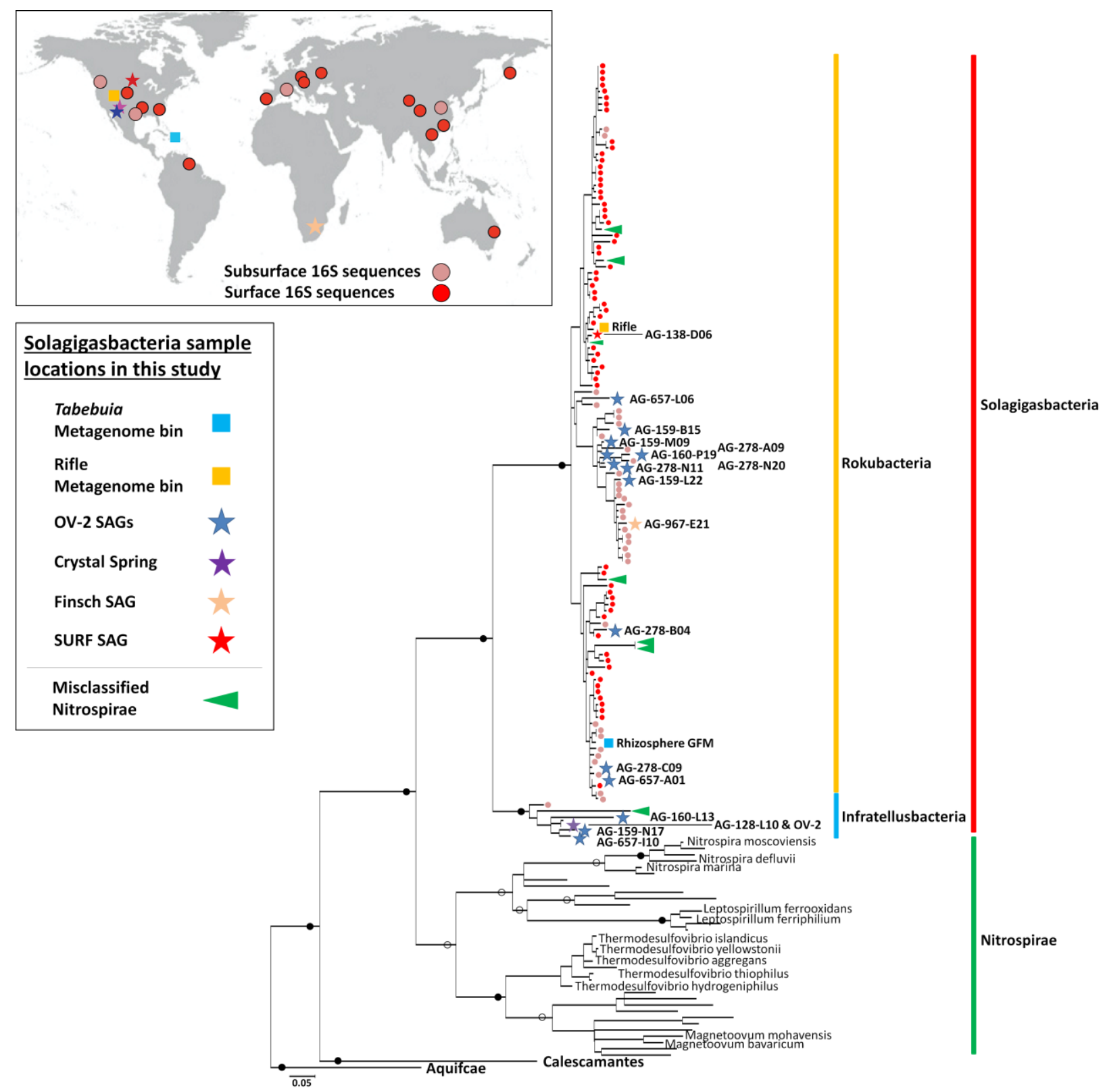

Figure 1. Maximum-likelihood phylogeny of the Solagigasbacteria (red line), based on partial 16S rRNA gene sequences ( $\sim 600 \mathrm{bps}$ in length). Included are NCBI sequences with $\geq 85 \%$ nucleotide identity to Solagigasbacteria SAGs. The Rokubacteria are demarcated by a dark orange line, and the Infratellusbacteria are demarcated by a light blue line. Nitrospirae genome 16S rRNA gene sequences representing classified genera and sequences misclassified as Nitrospirae are demarcated by a green vertical bar and green arrows, respectively. 16S rRNA gene sequence from previous surface (red circles), and subsurface (tan circles) studies are also indicated at the terminal branch of each sequence. Map insert (upper left) shows geographic distribution of reported Solagigasbacteria 16S rRNA gene sequences from past surface (red circles), and subsurface (tan circles) studies. Sequence identifiers are reported in Supplemental Figure 3. Previously sampled subsurface sites from Puerto Rico and Nevada where Solagigasbacteria 16S rRNA genes sequences were identified are not shown in Figure 1 insert due to space constants. Stars indicate Solagigasbacteria SAGs (blue = OV-2, purple = Crystal Spring; $\tan =$ Finsch mine; and red $=$ SURF) and squares indicate metagenome bins (light blue = Tabebuia ; orange $=$ Rifle site), and color corresponds to site SAGs were isolated from (left; also see Supplemental Figure 2). All SAGs and metagenome bins that contained a 16S rRNA gene are included in the phylogeny. Full circles indicate bootstrap values $>90 \%$; open circles indicate boot-strap values $>70 \%$. Scale bar represents 0.05 nucleotide substitutions per site. 


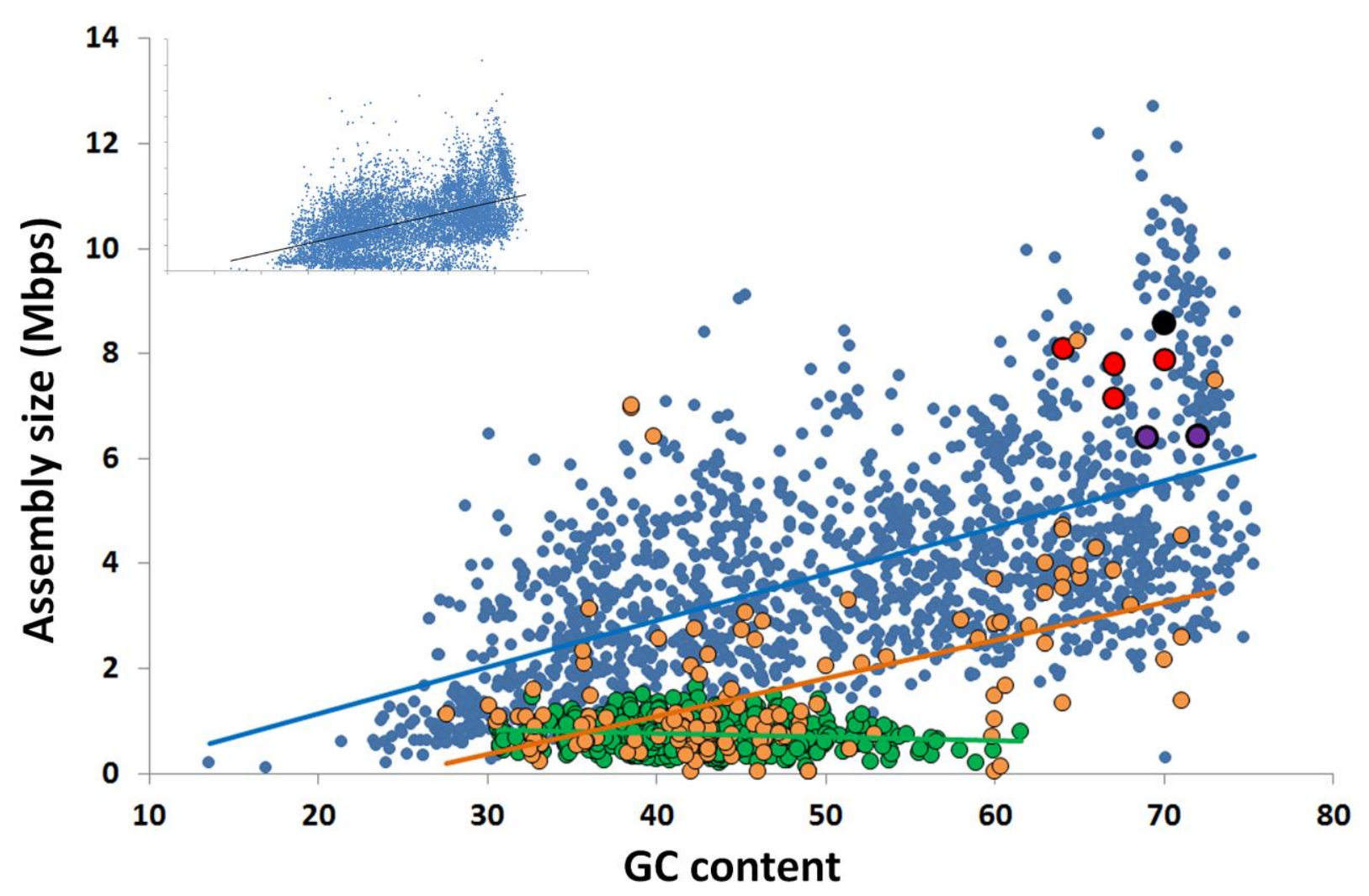

Figure 2. Correlation between the $\mathrm{G}+\mathrm{C}$ content and genome size among all finished bacterial genomes in IMG (blue; $\mathrm{R}^{2}=0.35$ ), The 4 most complete Solagigasbacteria SAGs from OV-2 (red), metagenome bins from OV-2 (bin 8) and Puerto Rican Tabebuia rhizosphere that contain a 16S rRNA gene (purple), and the Rifle metagenome bin that contains a 16S rRNA gene (black) (also see Supplemental Table 3). Also displayed are the estimated genome sizes for Candidate Phyla Radiation $(\mathrm{CPR})$ genomes (green; $\mathrm{R}^{2}=0.02$ ) and candidate phyla genomes not a part of the CPR lineage (orange; $R^{2}=0.25$ ). The insert contains all genomes in IMG, including all partial SAG and metagenome bins from all bacterial phyla $\left(\mathrm{R}^{2}=0.35\right)$. 

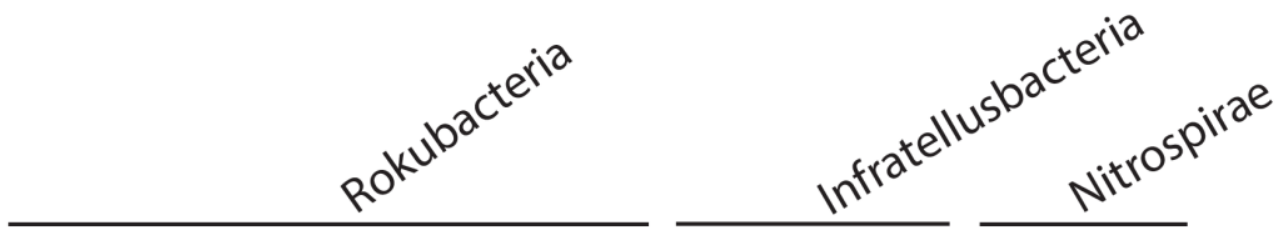

AG-278-N20

AG-278-N11

AG-160-P19

AG-159-M09

AG-967-E21

AG-159-L22

AG-657-L06

Rhizosphere bin

AG-278-B04

AG-278-C09

Rifle bin

OV-2 bin 8

AG-128-L10

AG-657-110

AG-159-N17

AG-159-L13

N. defluvii

L. ferrooxidans

T. islandicus

M. bavaricum

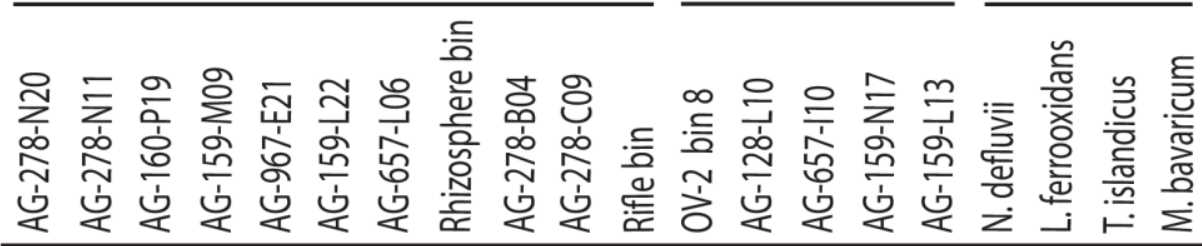

Figure 3. Average amino acid identity (AAI) for shared proteins among more complete Solagigasbacteria single amplified genomes (SAGs) (>0.5 Mbps) and Solagigasbacteria metagenome bin8 and rhizosphere bin (indicated by asterisks in Table 1), and select genomes from the Nitrospirae phylum. Boxes indicate phylogenetically defined class-level lineages or above (also see Figure 1 and Supplemental Table 2). 


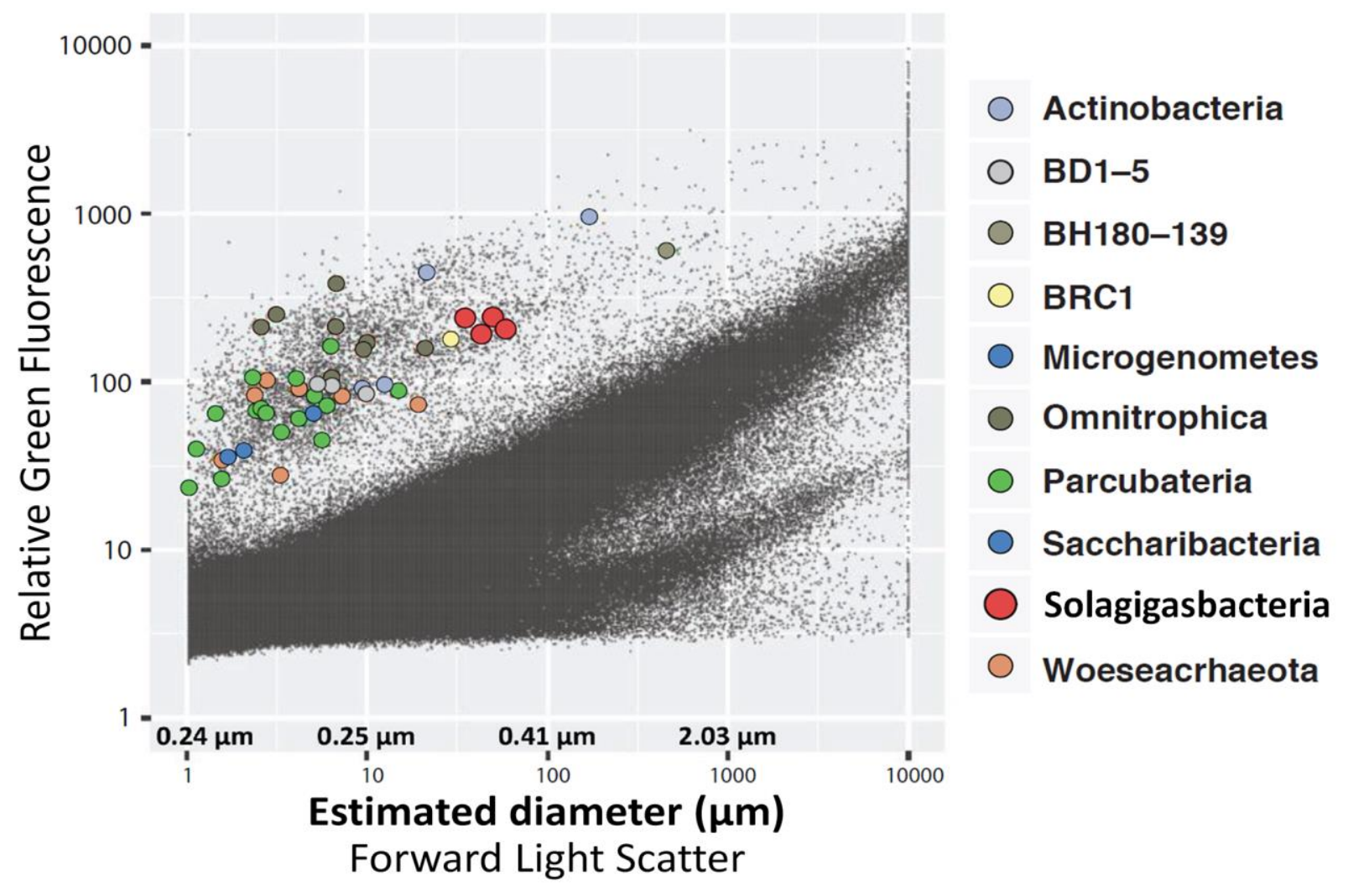

Figure 4. Optical properties and estimated diameters of cells sorted from the OV-2 sample that contained the largest $16 \mathrm{~S}$ rRNA gene. Black dots indicate all particles detected by the fluorescence-activated cell sorter. 


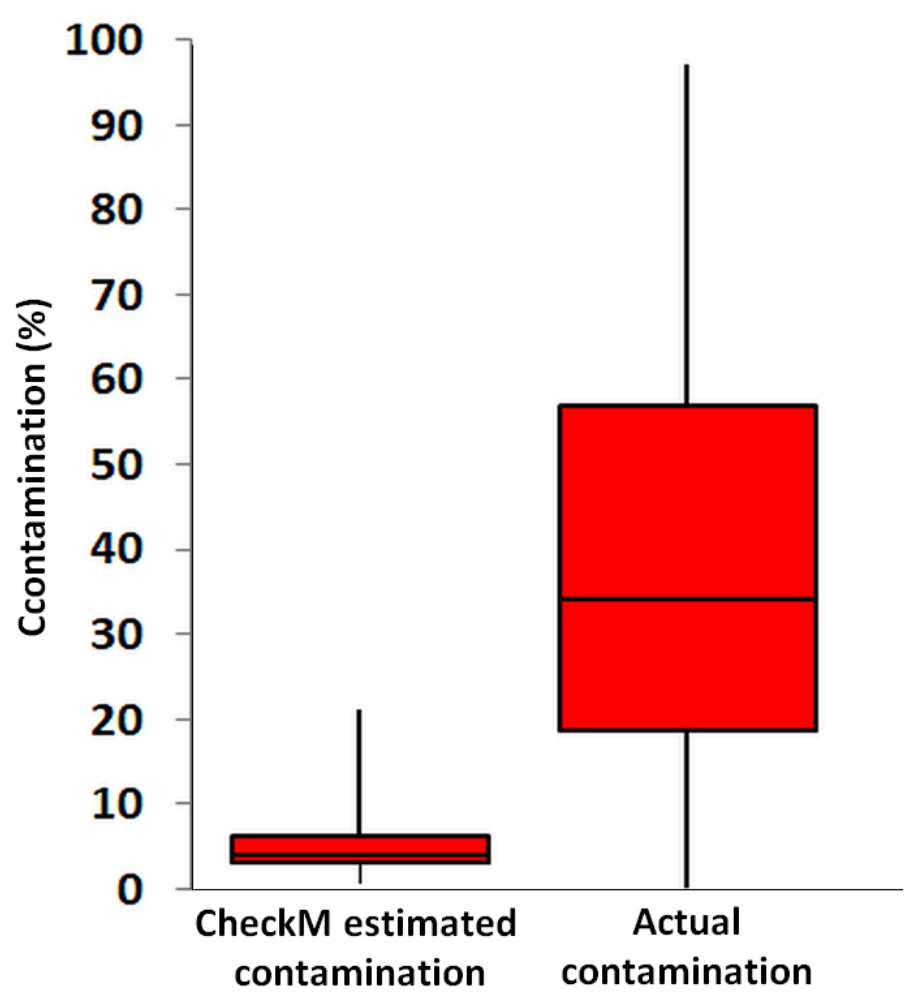

Figure 5. Contamination predicted by checkM software from all pairwise SAG combinations 705 across the class-level lineages Rokubacteria and Infratellusbacteria (15 Rokubacteria and 4 Infratellusbacteria SAGs; 60 combinations total), compared to actual contamination calculated from all artificially combined SAGs. Two-sample t-test assuming equal variances was significant $(\mathrm{p}=<000.1)$. 
Table 1. Solagigasbacteria assembly statistics and predicted genome completeness. Asterisks indicate more complete genome 714 assemblies that were used in Figure 3.

\begin{tabular}{|c|c|c|c|c|c|c|c|c|c|c|}
\hline $\begin{array}{l}\text { Solagigasbacteria } \\
\text { SAGs }\end{array}$ & Class & Site & $\begin{array}{c}\text { Raw PE } \\
\text { reads } \\
(\text { millions })\end{array}$ & $\begin{array}{l}\text { Assembly } \\
\text { (Mbps) }\end{array}$ & $\begin{array}{c}\text { GC } \\
\%\end{array}$ & $\begin{array}{c}16 \mathrm{~S} \\
\text { rRNA+ }\end{array}$ & $\begin{array}{c}\text { Estimated } \\
\text { Genome } \\
\text { Completeness, } \\
\%\end{array}$ & $\begin{array}{l}\text { Predicted } \\
\text { genome } \\
\text { size- } \\
\text { Mbps }\end{array}$ & $\begin{array}{c}\% \\
\text { Contami- } \\
\text { nation }^{4}\end{array}$ & $\begin{array}{l}\text { Genome } \\
\text { quality }^{5}\end{array}$ \\
\hline *AD-967-E21 & Rokubacteria & Finsch & 13.7 & 1.52 & 66 & Yes & 30 & 5.1 & $<1$ & low \\
\hline *AG-128-L10 & Infratellusbacteria & $\mathrm{CS}^{1}$ & 13.2 & 1.89 & 70 & Yes & 24 & 7.9 & $<1$ & low \\
\hline AG-138-D06 & Rokubacteria & SURF $^{2}$ & 12.0 & 0.29 & 67 & Yes & 6 & 4.9 & 0 & low \\
\hline AG-657-A01 & Rokubacteria & $\mathrm{OV}-2$ & 12.2 & 0.40 & 68 & Yes & $<1$ & NA & 0 & low \\
\hline *AG-657-I10 & Infratellusbacteria & $\mathrm{OV}-2$ & 10.8 & 0.93 & 71 & Yes & $<1$ & NA & 0 & low \\
\hline *AG-657-L06 & Rokubacteria & $\mathrm{OV}-2$ & 7.7 & 1.03 & 65 & Yes & $<1$ & NA & 0 & low \\
\hline AG-159-B15 & Rokubacteria & $\mathrm{OV}-2$ & 8.8 & 0.05 & 64 & Yes & $<1$ & NA & 0 & low \\
\hline AG-159-G23 & Rokubacteria & $\mathrm{OV}-2$ & 7.6 & 0.40 & 64 & No & 5 & 8.1 & 0 & low \\
\hline *AG-159-L22 & Rokubacteria & $\mathrm{OV}-2$ & 8.4 & 0.69 & 65 & Yes & 16 & 4.3 & 0 & low \\
\hline *AG-159-M09 & Rokubacteria & $\mathrm{OV}-2$ & 7.5 & 0.93 & 65 & Yes & 4 & 22.2 & 0 & low \\
\hline AG-159-N17 & Infratellusbacteria & $\mathrm{OV}-2$ & 9.8 & 0.41 & 67 & Yes & $<1$ & NA & 0 & low \\
\hline AG-159-P01 & Rokubacteria & $\mathrm{OV}-2$ & 5.1 & 0.07 & 65 & No & $<1$ & NA & 0 & low \\
\hline AG-160-L13 & Infratellusbacteria & $\mathrm{OV}-2$ & 7.0 & 0.41 & 64 & Yes & 3 & 13.7 & 0 & low \\
\hline *AG-160-P19 & Rokubacteria & $\mathrm{OV}-2$ & 8.2 & 1.38 & 64 & Yes & 27 & 5.1 & $<1$ & low \\
\hline AG-278-A09 & Rokubacteria & $\mathrm{OV}-2$ & 0.08 & 0.24 & 65 & Yes & 4 & 6.1 & 0 & low \\
\hline *AG-278-B04 & Rokubacteria & $\mathrm{OV}-2$ & 8.4 & 2.61 & 69 & Yes & 32 & 8.2 & $<1$ & low \\
\hline *AG-278-C09 & Rokubacteria & $\mathrm{OV}-2$ & 8.5 & 1.68 & 68 & Yes & 18 & 9.21 & 0 & low \\
\hline *AG-278-N11 & Rokubacteria & $\mathrm{OV}-2$ & 7.4 & 1.15 & 67 & Yes & 15 & 7.8 & 0 & low \\
\hline *AG-278-N20 & Rokubacteria & $\mathrm{OV}-2$ & 0.16 & 2.86 & 67 & Yes & 40 & 7.2 & 0 & low \\
\hline \multicolumn{11}{|l|}{$\begin{array}{l}\text { Solagigasbacteria } \\
\text { metagenome bins }\end{array}$} \\
\hline$* O V-2$ bin8 ${ }^{3}$ & Infratellusbacteria & $\mathrm{OV}-2$ & - & 5.69 & 72 & yes & 89 & 6.4 & 2 & Medium \\
\hline *Rhizosphere bin $^{3}$ & Rokubacteria & PR & - & 4.01 & 69 & yes & 63 & 6.4 & 1 & Low \\
\hline OV-2 bin 1 & unknown & $\mathrm{OV}-2$ & - & 11.84 & 62 & no & 100 & 11.8 & 332 & NA \\
\hline OV-2 bin2 & unknown & $\mathrm{OV}-2$ & - & 11.68 & 69 & no & 100 & 11.6 & 175 & NA \\
\hline OV-2 bin6 & unknown & $\mathrm{OV}-2$ & - & 6.06 & 69 & no & 73 & 8.3 & 59 & NA \\
\hline OV-2 bin9 & unknown & $\mathrm{OV}-2$ & - & 5.05 & 71 & no & 78 & 6.5 & 34 & NA \\
\hline OV-2 bin11 & unknown & $\mathrm{OV}-2$ & - & 4.68 & 68 & no & 98 & 4.8 & 153 & NA \\
\hline OV-2 bin 43 & unknown & $\mathrm{OV}-2$ & - & 1.32 & 72 & no & 14 & 9.5 & 1 & Low \\
\hline
\end{tabular}

$715 \quad{ }^{1,2}$ Crystal Spring, Nevada and Sanford Underground Research Facility $(300 \mathrm{~m})$

$716{ }^{3}$ Metagenome bins from OV-2 and Tabebuia rhizosphere sample taken in Puerto Rico.

$717{ }^{4}$ Estimated with checkM

$718{ }^{5}$ Genome quality reported according to Bowers et al., 2017. 